\title{
THE EFFECTS OF TASK LOAD AND VEHICLE HETEROGENEITY ON PERFORMANCE IN THE MULTIPLE-VEHICLE TRACKING TASK
}

\author{
Martin J. Lochner ${ }^{1}$ \& Lana M. Trick ${ }^{2}$ \\ ${ }^{1}$ Commonwealth Scientific and Industrial Research Organization, Hobart, Tasmania, Australia \\ ${ }^{2}$ University of Guelph, Guelph, Ontario, Canada \\ E-mails: martin.lochner@csiro.au and ltrick@uoguelph.ca
}

\begin{abstract}
Summary: When crossing traffic at busy intersections, drivers must keep track of the changing positions of cyclists, pedestrians and other vehicles to avoid collision. Multiple-object tracking is the ability to monitor the positions of a number of selected moving objects (targets) among others (distractors) in a complex scene. Most young adults can track 3-5 items at once but older adults cannot track as many, a finding that may partially explain older drivers' increased risk at intersections. Because tracking represents an important component of driving, a variant of the multiple-object tracking task called multiple-vehicle was created to measure tracking performance in a driving simulator. However, it is unclear whether tracking while driving works the same as tracking carried out on its own. Laboratory studies suggest that tracking improves when the moving items are heterogeneous, and on the road, it is far more typical that vehicles differ from one another rather than being all the same. Drivers were given the task of tracking the positions of 4 vehicles in a field of 8 on a highway, and the effects of task load (tracking alone, tracking while driving) on tracking performance were measured as a function of whether the target and distractor vehicles were homogeneous. Steering and headway maintenance variability were also assessed. The results indicated that heterogeneity only enabled better tracking when drivers were tracking in isolation. Heterogeneity had no significant effect on tracking when participants were tracking while driving though it did significantly reduce their steering variability.
\end{abstract}

\section{OBJECTIVES}

Multiple-object tracking is the ability to monitor and report the positions of a small number of moving items at once among other moving items (Pylyshyn, 1989). The ability to keep track of the changing positions of other road users (drivers, pedestrians, cyclists) would seem to be fundamental to safe driving but at this point, multiple-object tracking has rarely been studied in the driving literature. Although this task may have some promise in predicting driver performance, at this point it is not clear whether tracking as carried out in the laboratory works the same as it does while driving. In this study we examined the role of object heterogeneity in tracking performance as measured in a driving simulator.

In the multiple-object tracking task, participants are faced with a number of identical objects (e.g. 8), a subset of which flash to indicate that they are targets (e.g. 1-4 items). The objects then all become identical once more, and go into rapid independent movement for a period of time, after which the participants indicate the positions of items that were targets. Pylyshyn (1989) found that young adults could accurately track up to 3-5 targets, even though the items were moving too rapidly for the participants to move their eyes, or even their attentional focus from one item to another (Horowitz et al. 2004). Although the ability to simultaneously keep track of 
the positions of a small number of items at once seems remarkable, 25 years of research suggest that it is a robust finding. People can even track with accuracy when items momentarily occlude one another or pass behind other objects that occlude them (see Horowitz et al. 2006).

To date, there have been very few studies that look at multiple-object tracking as it relates to driving, though there are a variety of situations where the ability to keep track of the positions of several moving road users at once might be useful, such as when a driver is negotiating a busy multilane highway, passing, or merging in traffic. The clearest case for the need for multipleobject tracking occurs at complex un-signaled intersections where drivers have to cross traffic, avoiding vehicles, pedestrians, and cyclists. These types of intersection are indeed associated with higher risk, especially among older drivers (e.g., Eck \& Winn, 2002; Skyvving, Berg, \& Laflamme, 2009), and recently there have been several investigations of performance at intersections. These studies investigate eye movements (e.g. Dukic \& Broberg, 2012; Romoser, Pollatsek, Fisher, \& Williams, 2013) differentiating the way that older and younger drivers move their eyes at intersections. This research has made important contributions, but it does not tell the whole story. Eye movement analysis tells where the driver is directing the foveal area of the eyes (the part of the eye best suited for picking up fine detail), but it has long been known that people may attend to objects that they are not foveating (e.g. Posner, 1980). Conversely, the notorious "looked-but-failed-to-see" collision demonstrates that drivers may foveate objects and yet fail to attend them (e.g., White \& Caird, 2010). Furthermore, Hardiess, Hansmann-Roth, and Mallot (2013) found that there was no difference in the eye movement patterns between those who make correct and incorrect decisions about when they can safely cross at multilane intersections, which led the authors to conclude that driver safety may be more related to the ability to represent the positions of multiple vehicles at once rather than eye movements per se.

In contrast, although people typically perform the tracking task without making eye movements, the task definitely requires the ability to represent the positions of multiple moving objects at once. In fact, Pylyshyn (1989) argued that multiple-object tracking requires an ability that is a prerequisite for accurate eye movements. Eye movements are relatively slow (the initiation of an eye movement often takes up to 100-250 ms) and if eye movements are made to foveate moving objects, there needs to be a way to ensure the fovea arrives at where the object is and not where it was. Pylyshyn proposed that the ability that serves as the basis for multiple-object tracking is fundamental to visual motor coordination given that objects are constantly moving and changing. There are individual differences in tracking performance though. At the one end of the continuum, there are those that seem to be able to track more things at once than others. In sports such as hockey, one of the things that may distinguish the best players from others that are merely average, is the ability to keep track of the puck, as well as the other players. There are now several studies that link multiple-object tracking to performance in sports such as hockey, football, and soccer (Faubert 2012, 2014). At the other end, there are those who cannot track as well as others. For example, with advanced age there are declines in the number of items that can be tracked (e.g. Trick, Perl \& Sethi 2005). These declines may be a factor in intersection collisions, and although a variety of measures have been used to predict performance in older drivers, a recent study included multiple-object tracking as one (e.g. Bowers et al. 2013). However, the problem with correlational evidence of this type is that if a relationship between tracking and driving performance exists, it is unclear whether it is because multiple-object 
tracking is actually used in driving or whether it just correlates with other abilities that are used in driving.

Lochner and Trick (2014) did the first study that incorporated multiple-object tracking into a driving task. Their goals were to find out whether it was possible to track while driving and determine whether there were any costs and benefits to tracking while driving. In this "multiplevehicle tracking" task, participants were required to track up to 4 vehicles in a field of up to 8 moving vehicles in a platoon of rapidly shifting vehicles on a multilane highway. Their results indicated that multiple-object tracking while driving was possible, but it had costs: increasing the number of vehicles to be tracked also increased steering and headway variability. These are aspects of driving thought to be under the control of Wicken's (2002) ambient attention, which is thought to govern the driver's position in space without making extensive demands on foveal information. Tracking also had benefits: drivers were up to 14-16\% more accurate at localizing the vehicle that changed (e.g. the position of the vehicle that braked) if it was a target rather than a distractor, and up to $250 \mathrm{~ms}$ faster making this localization decision. (Localization information is relevant if drivers have to make evasive maneuvers while driving.)

The classic tracking task involves monitoring positions of targets among identical distractors. On the road, drivers are rarely faced with a cohort of identical road users. In this study we looked at the effects of heterogeneity, investigating multiple-vehicle tracking as a function of task load (tracking alone, tracking while driving) and tracking set composition: all homogeneous, all heterogenous, and a "paired-4" condition where there was a target and a matching distractor vehicle in each of four colours (a type of heterogeneity that would not help distinguishing targets from distractors, Makovski \& Jiang, 2009). Given the danger that the participants might form a strategy of rehearsing the colour names of target vehicles, we also had half of the participants track while performing an articulatory suppression task (continually saying "the the the"). Articulatory suppression is used to prevent verbal rehearsal (Richardson \& Baddeley, 1975). If participants were using a verbal rehearsal strategy to memorize the target vehicle colours while tracking, heterogeneity should be of no benefit when drivers were required to perform the articulatory suppression task at the same time. Based on the laboratory research, we predicted that heterogeneity would improve multiple-vehicle tracking performance, and in particular, heterogeneous condition performance would be superior to that in the homogeneous and paired-4 conditions even under articulatory suppression conditions (Makovski \& Jiang, 2009). In light of evidence that shows that factors that make the multiple-vehicle tracking more difficult also increase headway and steering and variability (Lochner \& Trick, 2014), we also predicted steering and headway variability should be highest in the homogeneous and paired-4 conditions.

\section{METHOD}

\section{Participants}

48 licensed drivers from the University of Guelph took part in the study for course credit (17 females; mean (and SD) for age $=20.3$ (1.6); driving experience $=4.3$ (1.7) years. All had normal colour vision and acuity. 


\section{Apparatus and Stimuli}

A Drivesafety DS 600c fixed-base driving simulator with $300^{\circ}$ of visual display was used for testing. The simulation involved a straight section of 3-lane freeway with 8 vehicles in front of the driver. Each vehicle subtended between $1.9^{\circ}$ and $3.44^{\circ}$ visual angle, depending upon its position in the set. Vehicle colours were either homogenous (i.e., all one colour), heterogenous (all different colours), or "paired-4" (one target and distractor in each colour - see Figure 1). The vehicles changed lanes at a rate of approximately $3.4^{\circ}$ per second on average.

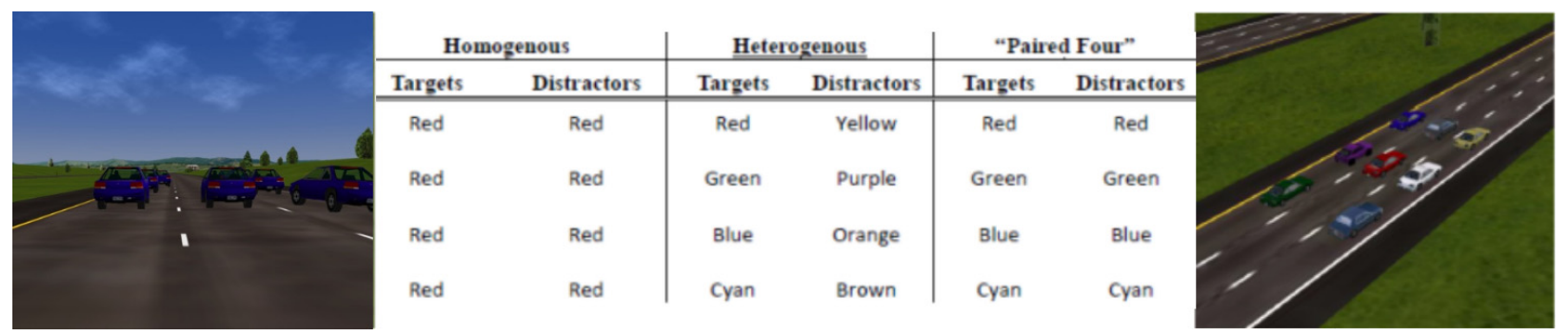

Figure 1. (Left side) Driver's view during a sample trial with blue vehicles in the Homogenous condition; (Middle) Example colour schemes for the Tracking Set Composition variable; (Right side) Isometric view of a trial in the Heterogeneous condition

\section{Design and Procedure}

Tracking Set (homogeneous, heterogeneous, paired-4) and Task Load (tracking alone, tracking while driving) were within subjects factors. Suppression Condition (articulatory suppression, no articulatory suppression) was between. That is, half of the drivers were required to track while carrying out the articulatory suppression task (repeating "the the the" at a steady rate) whereas the other half were not. The dependent measures were tracking accuracy, average headway, standard deviation of headway, and standard deviation of lateral position (SDLP).

Participants were required to track 4 target vehicles amidst 8 on a simulated 3-lane freeway. Each trial began with all vehicles stationary, after which the lead vehicles all accelerated to 80 $\mathrm{km} / \mathrm{hr}$. For drivers in the tracking alone condition, their vehicle accelerated automatically to keep the lead vehicles at a constant distance (no need for steering or accelerator control). Drivers in the tracking-while-driving condition had to accelerate to maintain a constant distance from the lead vehicles, and steer to control the lateral position of their car. Furthermore, half of the participants were required to perform articulatory suppression task. Once drivers attained the appropriate distance, a random 4 of the 8 vehicles flashed to indicate that they were targets. After the flashing stopped, the lead vehicles began switching lanes and this continued for 30 seconds. The speed of the lead cohort fluctuated during this period, but the average speed continued to be $80 \mathrm{kph}$. At the end of the trial, the simulation was paused and the participant checked off the position of the target vehicles on an answer sheet. The study began with 5 practice trials ( $\sim 5$ minutes). Task load was blocked and counterbalanced; tracking set was randomized. 


\section{RESULTS AND CONCLUSION}

Tracking accuracy was measured as the average percentage of correctly identified target vehicles (e.g. if the participant correctly identified 3 of the 4 target vehicles they were to track, their accuracy would be $3 / 4$ or $75 \%$ ). Overall tracking accuracy was $80 \%$. Based on the expected outcomes given the probability for sampling without replacement when tracking 4 vehicles in 8 , participants performed as well as would be expected if they were capable of tracking around 3 of the 4 vehicles they were required to track $(p<.05$ in one sample t-tests comparing the actual outcome with the expected outcome for guessing 2 of the 4 locations).

A $3 \times 2 \times 2$ mixed analysis of variance (ANOVA) was performed on the tracking accuracy data. Articulatory suppression had no significant effect $(p>.1)$, which suggests that participants were not using the strategy of mentally rehearsing the colour names of target vehicles. Consequently, the two articulation conditions were combined. As expected, the effect of tracking set was significant $(\mathrm{F}(2,94)=9.02, \mathrm{p}<.001, \eta 2=.16)$. Tests of means revealed that tracking accuracy was significantly better in the heterogenous than the homogenous and paired-4 conditions. Likewise, the predicted effect of task load emerged $(F(1,47)=16.06, p<.001, \eta 2=.27)$, with lower tracking accuracy when participants were required to drive while tracking. However, there was also a Tracking Set X Task Load interaction $(\mathrm{F}(2,94)=4.14, \mathrm{p}<.05, \eta 2=.08)$, indicating that the effects of the tracking set varied depending on task load (see Figure 2). In particular, the results indicated that tracking set composition had no effect when drivers were tracking while driving whereas it did when drivers were only tracking. This suggests that the ability to use item heterogeneity to augment tracking performance requires extra resources (Makovski \& Jiang, 2009). If driving requires the same limited capacity spatial resources, this might explain why driving while tracking negates any benefit of heterogeneity while articulatory suppression, which interferes substantially with verbal working memory, does not (Richardson \& Baddeley, 1975).

Interestingly, tracking set composition seemed to have different effects on headway maintenance and steering. Tracking set had no effect on average headway $(p>1)$, and though it did affect headway variability $(\mathrm{F}(2,94)=3.33, \mathrm{p}<.05$, partial $\eta 2=.07)$, the effects were not in the expected direction. Specifically, headway variability was lowest (and thus headway maintenance performance was best) in the paired-4 condition: a condition that should have had a higher headway variability given the poor tracking performance in that condition. However, tracking set did have the expected effect on steering variability $(F(2,94)=5.61, p<.05$, partial $\eta 2=.11)$, with significantly lower SDLP in the heterogeneous condition (the condition associated with the best tracking performance). See Figure 3.

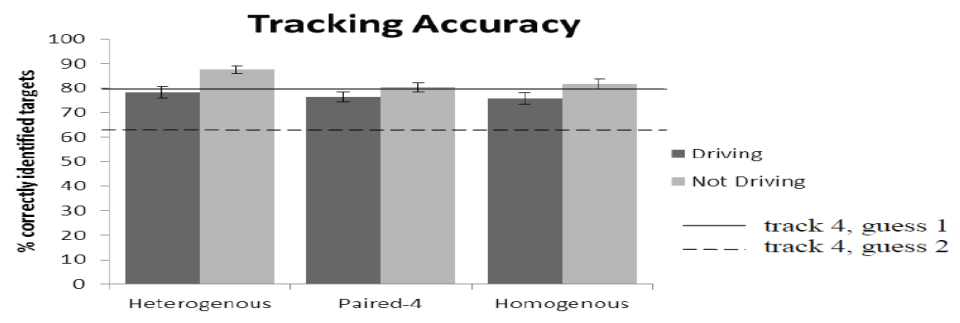

Figure 2. Tracking accuracy as a function of tracking set and task load (tracking alone, and tracking while driving). Solid and dotted lines indicate expected outcomes if participants were guessing the positions of 1 or 2 of the vehicles they were required to track 

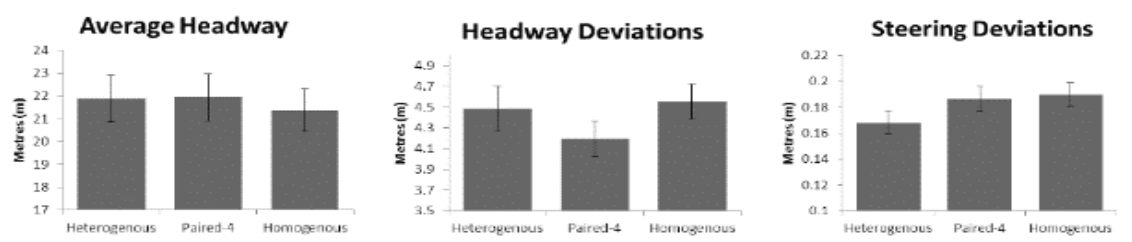

Figure 3. Driving metrics: Average headway, standard deviation of headway (headway deviation), and standard deviation of lateral position (SDLP or steering deviation) as a function of tracking set composition in the tracking while driving condition

This study represents one of the first investigations of multiple-object tracking in the context of driving. Ultimately our goal is to extend this paradigm to intersections but highway driving was the focus of this research because it permitted tracking to be studied with large numbers vehicles over extended periods of time. Admittedly, the task was somewhat exotic. We are rarely called upon to keep track of the positions of several specific vehicles in highway driving ("Follow those cars!"). However, this study showed that though item heterogeneity, particularly the type differentiates targets from distractors, can augment tracking performance, this advantage is negated if participants have to steer and control the vehicle's position while tracking. This finding is consistent with Pylyshyn's (1989) initial contention that the multiple-object tracking mechanism is purely spatial; keeping track of object properties requires additional resources. It is possible that this study underestimated the effects of heterogeneity insofar as it focused on vehicle colour. Colour is considered a primitive visual feature, one that "pops out" in visual search (Treisman \& Gelade, 1980) but vehicle colour is of little practical importance in driving. When driving, heterogeneity effects may be more notable in driving when items differ in category (e.g., pedestrian, cyclist, vehicle), but this question must be left to future investigations.

\section{ACKNOWLEDGEMENTS}

Grants to the second author from Auto21, the Canadian Foundation for Innovation, Ontario Innovation Trust, and Natural Sciences and Engineering Research Council of Canada funded this research. Author names are listed alphabetically (each author contributed equally to writing).

\section{REFERENCES}

Bowers, A.R., Anastasio, R.J., Sheldon, S.S., O’Connor, M.G., Hollis, A.M., Howe, P.D., \& Horowitz, T.D. (2013). Can we improve clinical prediction of at-risk older drivers? Accident Analysis and Prevention, 59(1), 537-547.

Dukic, T., \& Broberg, T. (2012). Older drivers' visual search behaviour at intersections. Transportation Research Part F, 15, 462-470.

Eck, R. W., \& Winn, G. (2002). Older-driver perception of problems at unsignalized intersections and divided highways. Transportation Research Record, 1818, 70-77.

Faubert, J. (2012). Perceptual-cognitive training of athletes. Journal of Clinical and Sport Psychology, 6, 85-102.

Faubert, J. (2014). Professional athletes have extraordinary skills for rapidly learning complex and neutral dynamic scenes. Scientific Reports, 3: 1154, DOI: 10.1038/srep01154 
Hardiess, G., Hansmann-Roth, S., \& Mallot, H.A. (2013). Gaze movements and spatial working memory in collision avoidance: A traffic intersection task. Frontiers in Behavioural Neuroscience, 7 (62), 1-13. doi: 10.3389/fnbeh.2013.00062

Horowitz, T.S., Birnkrant, R. S., Fencsik, D. E., Tran, L., \& Wolfe, J. M. (2006). How do we track invisible objects? Psychonomic Bulletin \& Review, 13(3), 516-523.

Horowitz, T.S., Holcombe, A.E., Wolfe, J.M., Arsenio, H.C., \& DiMase, J.S. (2004). Attentional pursuit is faster than attentional saccade. Journal of Vision, 4, 585-603.

Lochner, M.J. \& Trick, L.M. (2014). Multiple-object tracking while driving: The multiple-vehicle tracking task. Attention, Perception, and Psychophysics, 76, 2326-2345.

Makovski, T., \& Jiang, Y.V., (2009). The role of visual working memory in attentive tracking of unique objects. Journal of Experimental Psychology: Human Perception and Performance, 35 (6), 1687-1697.

Posner, M. I. (1980). Orienting of attention, Quarterly Journal of Psychology, 32, 3-25.

Pylyshyn, Z.W. (1989). The role of location indexes in spatial perception: A sketch of the FINST spatial-index model. Cognition, 32 (1), 65-97.

Richardson, J.T.E., \& Baddeley, A.D. (1975). The effect of articulatory suppression in free recall. Journal of Verbal Learning and Verbal Behaviour, 14(6), 623-629.

Romoser, M.R.E., Pollatsek, A., Fisher, D.L., \& Williams, C.C. (2013). Comparing the glance patterns of older versus younger experienced drivers: Scanning for hazards while approaching and entering the intersection. Transportation Research Part F, 16, 104-116.

Skyving, M., Berg, H.Y., \& Laflamme, L. (2009). A pattern analysis of traffic crashes fatal to older drivers, Accident Analysis and Prevention, 41, 253-258.

Treisman, A., \& Gelade, G. (1980). A feature integration theory of attention. Cognitive Psychology, 12, 97-136.

Trick, L.M., Perl, T., \& Sethi, N. (2005). Age-related differences in multiple-object tracking. Journal of Gerontology: Series B, 60B (2), 102-105.

White, C.B., \& Caird, J.K. (2010). The blind date: The effects of change blindness, passenger conversation and gender on looked-but-failed-to-see (LBFTS) errors. Accident Analysis and Prevention, 42(6), 1822-1830.

Wickens, C. D. (2002). Multiple resources and performance prediction. Theoretical Issues in Ergonomics Science, 3 (2), 159-177. 